\title{
THE WIDE-FIELD INFRARED EXPLORER (WIRE) MISSION
}

\author{
D.L. SHUPE ${ }^{1}$, P.B. HACKING ${ }^{2}$, T. HERTER ${ }^{3}$, T.N. GAUTIER ${ }^{4}$, \\ P. GRAF ${ }^{5}$, C.J. LONSDALE ${ }^{1}$, G.J. STACEY ${ }^{3}$, S.H. MOSELEY ${ }^{6}$, \\ B.T. SOIFER ${ }^{7}$, M.W. WERNER ${ }^{4}$ AND J.R. HOUCK ${ }^{3}$ \\ ${ }^{1}$ Infrared Processing and Analysis Center, Pasadena, CA \\ ${ }^{2}$ Jamieson Science \&3 Engineering, Bethesda, MD \\ ${ }^{3}$ Cornell University, Department of Astronomy, Ithaca, NY \\ ${ }^{4}$ Jet Propulsion Laboratory, Pasadena, CA \\ ${ }^{5}$ Ball Technologies, Boulder, CO \\ ${ }^{6}$ Goddard Space Flight Center, Greenbelt, MD \\ ${ }^{7}$ California Institute of Technology, Pasadena, CA
}

\section{Introduction}

The Wide-Field Infrared Explorer (WIRE) (Schember et al. 1996 and references within) is a small spaceborne telescope specifically designed to study the evolution of starburst galaxies. This powerful astronomical instrument will be capable of detecting typical starburst galaxies at $z \sim 0.5$, ultraluminous infrared galaxies beyond a $z \sim 2$, and luminous protogalaxies beyond $z \sim 5$. The WIRE survey, to be conducted during a four month period in 1998 , will cover over $100 \mathrm{deg}^{2}$ of high Galactic latitude sky at $25 \mu \mathrm{m}$ and $12 \mu \mathrm{m}$.

WIRE was selected as the fifth in NASA's series of Small Explorer (SMEX) missions in August 1994. It was proposed in 1992 by a teaming partnership of the Jet Propulsion Laboratory (JPL), California Institute of Technology, and the Space Dynamics Laboratory (SDL), Utah State University. Launch is scheduled for September 1998.

\section{WIRE Primary Science}

The WIRE survey will detect primarily starburst galaxies, which emit most of their energy in the far-infared. The number of these faint sources at a given flux level depends on their as-yet-unknown evolutionary rate. The objective of WIRE is to answer the following three questions: (1) What 
fraction of the luminosity of the Universe at a redshift of 0.5 and beyond is due to starburst galaxies? (2) How fast and in what ways are starburst galaxies evolving? (3) Are luminous protogalaxies common at redshifts less than 3 ?

The WIRE survey will cover over $100 \mathrm{deg}^{2}$ of sky and detect sources 200-500 times fainter than the IRAS Faint Source Catalog at $25 \mu \mathrm{m}$ and 500-2000 times fainter at $12 \mu \mathrm{m}$. The resulting catalog, expected to contain at least 30,000 starburst galaxies, will reveal their evolutionary history out to $z \sim 0.5-1$ and the evolutionary history of extremely luminous galaxies beyond $z \sim 5$. This will be the first significant galaxy survey to probe these redshifts at far-infared wavelengths where extinction effects are small and where most of the luminosity of starburst galaxies, and possibly of the Universe, can be measured. WIRE will measure the $25 \mu \mathrm{m}-12 \mu \mathrm{m}$ color of detected sources, which is a powerful statistical luminosity indicator (Soifer \& Neugebauer 1991) as well as an effective means of distinguishing foreground stars.

\section{The WIRE Instrument}

The WIRE instrument is a cryogenically-cooled $30 \mathrm{~cm}$ Ritchey-Chrétien telescope system that illuminates two $128 \times 128 \mathrm{Si}$ :As infrared detector arrays. A two-stage solid hydrogen cryostat maintains the optics colder than $19 \mathrm{~K}$ and the detector arrays below $7.5 \mathrm{~K}$. The optical system consists of the telescope primary and secondary mirrors, a dichroic beamsplitter, one optical passband filter, and baffles. The two channels of the instrument cover broad bands centered near $12 \mu \mathrm{m}$ and $25 \mu \mathrm{m}$; the $25 \mu \mathrm{m}$ band is the primary one for detecting starburst galaxies. The pixels are 15.5 arcsec on a side, providing a $33 \times 33 \mathrm{arcmin}^{2}$ field of view in each passband. The FWHM beam size in coadded images will be about 26 arcsec at $25 \mu \mathrm{m}$ and $22 \operatorname{arcsec}$ at $12 \mu \mathrm{m}$. The instrument contains no moving parts.

\section{Survey and Observing Strategy}

The WIRE survey will consist of three parts. The moderate-depth survey is designed to maximize the detection of distant protogalaxies. $60 \%$ of the survey time will be spent on this survey, covering hundreds of square degrees, with 15 to 50 minutes total exposure time on each WIRE field. $30 \%$ of the survey time will be spent on the deep survey, with a total integration time of several hours per field, set by the point at which confusion noise is equal to instrumental noise. The goal of this survey is to obtain a large sample with the largest lookback time at a given luminosity, which will require covering tens of square degrees to this depth. Finally, the ultradeep survey will use about $10 \%$ of the survey time early in the mission to 
observe a few WIRE fields for 24 hours or more total exposure time, to measure the confusion distribution.

To reach such large cumulative exposure times, WIRE will use a stareand-dither observing technique. During a ten minute orbit segment when the target field is near the zenith, the instrument will record several short $(44 \mathrm{sec})$ exposures, each separated by a small dither. This technique will allow accurate subtraction of the background from the data. Target fields will be reacquired on subsequent orbits to accumulate sufficient exposure time and to allow detection of moving or variable sources.

The sensitivity of the deep survey will be limited by source confusion and hence depends on the rate of starburst galaxy evolution. Expected sensitivities are listed in Tables 1 and 2 for two evolution cases. The $12 \mu \mathrm{m}$ sensitivity will be 1.5 to 3 times lower (in mJy at $12 \mu \mathrm{m}$ ) than the limits tabulated for $25 \mu \mathrm{m}$.

TABLE 1. WIRE Sensitivity for $(1+z)^{1.7}$ Density Evolution

\begin{tabular}{lccc}
\hline Survey & Sky Coverage & $25 \mu \mathrm{m}$ Flux limit $(5 \sigma)$ & \# of sources \\
\hline Moderate Depth & $400 \mathrm{deg}^{2}$ & $1 \mathrm{mJy}$ & $>90,000$ \\
Deep & $20 \mathrm{deg}^{2}$ & $0.4 \mathrm{mJy}$ & 17,000 \\
Ultra-deep & $1 \mathrm{deg}^{2}$ & $0.28 \mathrm{mJy}$ & 1,000 \\
\hline
\end{tabular}

TABLE 2. WIRE Sensitivity for No Evolution

\begin{tabular}{lccc}
\hline Survey & Sky Coverage & $25 \mu \mathrm{m}$ Flux limit $(5 \sigma)$ & \# of sources \\
\hline Moderate Depth & $145 \mathrm{deg}^{2}$ & $0.65 \mathrm{mJy}$ & $>35,000$ \\
Deep & $7 \mathrm{deg}^{2}$ & $0.27 \mathrm{mJy}$ & 6,000 \\
Ultra-deep & $0.5 \mathrm{deg}^{2}$ & $0.22 \mathrm{mJy}$ & 500 \\
\hline
\end{tabular}

\section{References}

Schember, H., Kemp, J., Ames, H., Hacking, P., Herter, T., Everett, D., Sparr, L., and Fafaul, B., 1996, Infrared Technology and Applications XXII, SPIE Proceedings 2744, in press.

Soifer, B.T., \& Neugebauer, G., 1991 Astron.J. 101, 354. 